ORIGINAL ARTICLE

\title{
Ground reaction forces associated with an effective elementary school based jumping intervention
}

\author{
H McKay, G Tsang, A Heinonen, K MacKelvie, D Sanderson, K M Khan
}

Br J Sports Med 2005;39:10-14. doi: 10.1136/bjsm.2003.008615

See end of article for authors' affiliations

Correspondence to: Dr McKay, Department of Family Practice and Orthopaedics, University of British Columbia, Suite 211, 2150 Western Parkway, Vancouver V6T 1V6, Canada; mcKayH@ interchange.ubc.ca

Accepted 6 January 2004
Background: Mechanical loading during childhood plays a critical role in normal growth and development of the skeleton. Ground reaction forces (GRFs) may provide a surrogate measure for the strain experienced by bone on landing and at take off. However, there appear to be no paediatric studies that assess GRFs across a variety of loading activities.

Objectives: To measure biomechanical variables in commonly performed childhood activities used in an elementary physical education intervention study which augmented bone health in boys and girls.

Methods: Maximal GFR, maximal rates of force, and time to maximum force were measured for 12 different jumping activities on a force platform. The jumps measured were drop jumps from 10, 30, and $50 \mathrm{~cm}$, all followed by a plyometric jump, submaximal and maximal jumping jacks, alternating feet jump, counter movement jumps, and side to side jumps over 10 and $20 \mathrm{~cm}$ foam barriers. The subjects were 70 children (36 boys and 34 girls), 8.3-11.7 years old.

Results: Subjects ranged in height from 128.4 to $172.6 \mathrm{~cm}$ and had a mass of 25.0-57.0 kg. Mean (SD) for vertical jump was $24.2(5.5) \mathrm{cm}$ and $135.2(16.6) \mathrm{cm}$ for standing long jump. The children engaged in loaded physical activity 5.7 (5.3) hours a week, on average. The highest mean maximal GRFs, normalised for body weight (BW), were generated from the plyometric portion of the drop jumps and the counter movement jump (about 5 times BW) compared with 3.5 times BW for jumping jacks. Similarly, the highest rates of change in force were 514 times $\mathrm{BW} / \mathrm{s}$ for the drop jump from $10 \mathrm{~cm}$ and 493 times $\mathrm{BW} / \mathrm{s}$ for the counter movement jump.

Conclusions: Simple jumps requiring minimal equipment produce GRFs of 3.5-5 times BW and rates of force of around 500 times $\mathrm{BW} / \mathrm{s}$. As children appear to attenuate higher impact forces when jumping from increased heights, it cannot be assumed that merely increasing the height of the jump will necessarily "progress" the exercise intervention.
C hildhood is a key time to optimise bone mass and strength. ${ }^{12}$ Exercise intervention studies in children have successfully increased bone mass ${ }^{1-4}$ and structure, ${ }^{5}$ but the optimal amount and precise form of the physical activity stimulus necessary to achieve these results remains poorly characterised. Animal studies ${ }^{6-9}$ suggest that high or unusual strains increase bone strength by increasing bone mass, size, and area moment of inertia. Judex and colleagues ${ }^{10}$ elegantly showed that immature bone is sensitive to high strain rates.

A few pioneering studies in humans measured bone strain in vivo during physical activities that included walking, running, and drop jumps from various heights. ${ }^{11-14}$ Of interest was the finding that higher impact exercise (drop jumps from greater heights) did not cause higher tibial strain rates. ${ }^{11}$ Whether this is also the case in children remains unknown. Paediatric studies have not included in vivo strain gauge measurements. In 13 prepubertal children, Bauer et al ${ }^{15}$ measured ground reaction forces (GRFs) and force rate of change for a particular exercise: a drop jump from $61 \mathrm{~cm}$. Maximal GRF was 8.5 (2.2) times body weight (BW), and maximal rate of change of force was 472 times BW per second. There remains a need for further characterisation of activities that are osteogenic in children, particularly exercises other than the $61 \mathrm{~cm}$ drop jump characterised by Bauer et al. ${ }^{15}$ This will allow future optimisation of exercise programmes as well as the design of novel effective physical activity interventions to increase peak bone mass.

Because there have been few reports of the GRFs associated with a broad range of activities in children, we assessed GRFs and rate of change of force associated with a variety of jumps in a high impact loading intervention. We aimed also to compare results obtained from boys and girls. These activities formed part of the Healthy Bones II trial which significantly improved bone health through a simple modification of an elementary school physical education programme. $^{216}$

\section{METHODS}

Study design

We conducted a cross sectional study that assessed biomechanical variables for 12 types of jump performed by children within elementary school physical education. These jumps comprised part of a physical education curriculum that proved effective for improving bone mass and strength in boys and girls across both an eight month and a 20 month randomised controlled intervention trial. ${ }^{2} 1617$

\section{Subjects}

Subjects were grades 4,5 , and 6 students (8.3-11.7 years) attending elementary school in the Richmond School District in British Columbia, Canada. Seventy healthy children (36 boys, 34 girls) who had volunteered to participate in a separate high impact loading intervention study $(\mathrm{n}=383)$ were randomly selected for the present study. Subjects were screened for musculoskeletal problems, metabolic disorders, or the use of drugs that might affect balance or strength.

Abbreviations: $A F$, alternating feet; $B W$, body weight; $C M J$, counter movement jump; DJ, drop jump; GRF, ground reaction force; $J_{\text {max, }}$ maximal jumping jacks; JJ Jubmax, submaximal jumping jacks; $\mathrm{PJ}$, plyometric jump; RF, rate of change of the force; SS, side to side 
Table 1 Descriptive statistics for boys, girls, and the entire sample

\begin{tabular}{|c|c|c|c|c|}
\hline & Boys $(n=36)$ & Girls $(n=34)$ & Total $(n=70)$ & $95 \% \mathrm{Cl}$ \\
\hline Age (years) & $10.2(0.7)$ & $10.2(0.8)$ & $10.2(0.7)$ & (10.0 to 10.4$)$ \\
\hline Height $(\mathrm{cm})$ & $142.6(8.1)$ & $144.7(8.4)$ & $143.6(8.3)$ & (141.7 to 145.5 ) \\
\hline Mass $(\mathrm{kg})$ & $36.2(7.5)$ & $36.7(7.7)$ & $36.4(7.5)$ & (34.6 to 38.2 ) \\
\hline Vertical jump (cm) & $25.8(5.3)$ & $22.6(5.3)$ & $24.2(5.5)$ & (22.9 to 25.6$)$ \\
\hline Long jump $(\mathrm{cm})$ & $139.9(16.6)$ & $130.3(15.3)$ & $135.2(16.6)$ & (131.0 to 139.4$)$ \\
\hline Total physical activity (score/5) & $3.0(0.6)$ & $3.0(0.6)$ & $3.0(0.6)$ & (2.8 to 3.1$)$ \\
\hline Loaded physical activity time (hours) & $5.6(5.7)$ & $5.9(4.6)$ & $5.7(5.3)$ & (4.5 to 7.0$)$ \\
\hline
\end{tabular}

Means (SD) for age, height, and total body mass are presented for the group (table 1). We obtained written informed consent from all subjects and their parents before data collection. Ethics approval was obtained from the Richmond School Board and the University of British Columbia clinical research ethics board.

\section{Questionnaires}

\section{Physical activity}

We used the physical activity questionnaire for children (PAQ-C), a validated and reliable instrument that we have used extensively. ${ }^{18} \mathrm{~A}$ "load" score was derived from times in the past week that the child reportedly engaged in weight bearing activities (hours/week). These data were included as possible determinants of a "soft" landing technique, which, in turn, has been associated with magnitude of GRF. ${ }^{19}$

\section{Maturity}

Pubertal development was evaluated by self assessment of breast (girls) and pubic hair (boys), using a standard approach by the method of Tanner. ${ }^{20}$ We and others have used this assessment method in previous studies. ${ }^{1821}$ Self assessment of maturity is strongly correlated with staging assigned by an endocrinologist ${ }^{22}$ and is the only acceptable method suitable to assess children in a school based setting. As with the physical activity data, maturational data were included as possible determinants of landing techniques that may be associated with attenuation of GRF.

\section{Anthropometry and lower limb dynamic power}

Stretch stature (without shoes) and sitting height were measured to the nearest $0.1 \mathrm{~cm}$ with a mounted wall stadiometer using standard protocol. Mass was measured on the force platform within $0.1 \mathrm{~kg}$. Measurements were taken twice unless values were more than $0.4 \mathrm{~cm}$ (height and sitting height) or $0.2 \mathrm{~kg}$ (for mass) apart, then a third measure was taken. The average of two values and the median of three values were used as the final value. To evaluate lower limb dynamic power, maximal height $(\mathrm{cm})$ for vertical jump and distance $(\mathrm{cm})$ for standing long jump were evaluated using standard procedures. ${ }^{23}$

\section{Description of the jumps}

We used standard instructions and demonstrated proper jump technique for every participant for each of 12 different types of jump. Each subject performed the following jumps in random order: drop jump from $10 \mathrm{~cm}\left(\mathrm{DJ}_{10}\right)$, drop jump from $30 \mathrm{~cm}\left(\mathrm{DJ}_{30}\right)$, drop jump from $50 \mathrm{~cm}\left(\mathrm{DJ}_{50}\right)$, the aforementioned DJs followed by a maximal effort plyometric jump on landing $\left(\mathrm{PJ}_{10}, \mathrm{PJ}_{30}, \mathrm{PJ}_{50}\right)$, maximal jumping jacks $\left(\mathrm{JJ}_{\max }\right)$, submaximal jumping jacks $\left(\mathrm{JJ}_{\text {submax }}\right)$, alternating feet $(\mathrm{AF})$, side to side jumps over $10 \mathrm{~cm}\left(\mathrm{SS}_{10}\right)$ and $20 \mathrm{~cm}\left(\mathrm{SS}_{20}\right)$ foam barriers, and a counter movement jump (CMJ). A list of jumps is provided in the footnote to table 2 .

DJs were two foot landing drops down from a box of the aforementioned heights followed by a maximal jump. PJs were maximal effort two foot take off and landing jumps performed immediately after the DJ landing. JJs were performed with the subject's feet straddling the sides of the force platform and coming together on to the force platform, repeatedly. SS jumps began with feet together on the force platform, hands on hips and followed by a sideways two foot jump over a foam barrier. For CMJs the subject stood stationary, hands on hips, and then bent the knees rapidly (counter movement), and extended them as they jumped as high as possible. AF jumps were single foot landing jumps off of, and with the opposing foot on to, the force platform. The children performed the jumps in a series of three to five, except for the DJ, PJ and CMJ, which were performed only once.

Table 2 Maximal force and maximal rate of change in force for the 12 jumps

\begin{tabular}{|c|c|c|c|c|}
\hline \multirow[b]{2}{*}{ Type of jump } & \multicolumn{2}{|c|}{ Maximal force (BW) } & \multicolumn{2}{|c|}{ Maximal rate of change in force (BW/s) } \\
\hline & Mean (SD) & $95 \% \mathrm{Cl}$ & Mean (SD) & $95 \% \mathrm{Cl}$ \\
\hline CMJ & $5.3(1.6)$ & (4.9 to 5.8$)$ & $493(306)$ & (422 to 565$)$ \\
\hline $\mathrm{DJ}_{10}$ & $3.2(1.1)$ & $(3.0$ to 3.5$)$ & $241(198)$ & (194 to 287 ) \\
\hline $\mathrm{DJ}_{30}$ & $3.9(1.2)$ & (3.6 to 4.1 ) & $299(203)$ & (251 to 347 ) \\
\hline $\mathrm{DJ}_{50}$ & $4.7(1.7)$ & $(4.3$ to 5.1$)$ & $388(281)$ & (323 to 454 ) \\
\hline $\mathrm{PJ}_{10}$ & $5.5(2.0)$ & $(5.1$ to 6.0$)$ & $514(347)$ & (433 to 595 ) \\
\hline $\mathrm{PJ}_{30}$ & $5.2(1.5)$ & $(4.8$ to 5.5$)$ & $440(263)$ & (378 to 502 ) \\
\hline $\mathrm{PJ}_{50}$ & $5.4(1.6)$ & (5.1 to 5.8$)$ & $457(257)$ & (397 to 517 ) \\
\hline$J_{\text {submax }}$ & $3.5(0.5)$ & (3.5 to 3.6$)$ & $160(74)$ & (143 to 177$)$ \\
\hline$J J_{\max }$ & $3.4(0.4)$ & (3.3 to 3.6 ) & 211 (959) & (188 to 233 ) \\
\hline $\mathrm{AF}$ & $2.1(0.3)$ & (2.1 to 2.2 ) & $126(59)$ & (112 to 140 ) \\
\hline $\mathrm{SS}_{10}$ & $3.7(0.6)$ & (3.6 to 3.9 ) & $229(75)$ & (211 to 246 ) \\
\hline $\mathrm{SS}_{20}$ & $3.8(0.6)$ & (3.7 to 4.0 ) & 251 (99) & (228 to 274 ) \\
\hline
\end{tabular}

Values for the entire sample are shown ( $\mathrm{n}=70,36$ boys).

BW, body weight; Cl, confidence interval; CMJ, Counter movement jump; DJ $J_{10}$, drop jump $10 \mathrm{~cm}$; DJ 30 , drop jump $30 \mathrm{~cm}$; DJ 50 , drop jump $50 \mathrm{~cm}$; $\mathrm{PJ}_{10}$,

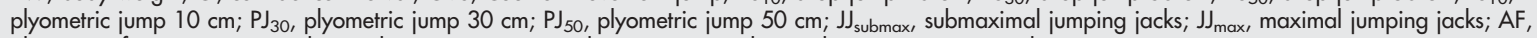
alternating feet jump; $\mathrm{SS}_{10}$, side to side jumps over $10 \mathrm{~cm}$ barrier; $\mathrm{SS}_{20}$, side to side jumps over $20 \mathrm{~cm}$ barrier. 


\section{GRF measurement and data processing}

GRFs for each jump were measured on a $40 \times 60 \mathrm{~cm}$ Kistler 9251A multicomponent force platform (Winterthur, Switzerland). Data were sampled for eight seconds at $300 \mathrm{~Hz}$ by an analogue/digital board (DT2821; Data Translation, Marlboro, Massachusetts, USA) and interfaced to the programme Peak Motus (Peak Performance Technology, Englewood, Colorado, USA). The platform was mounted flush with the floor surface. Subjects wore their normal running shoes. Data were processed with software developed in house using Microsoft Excel. All force data were normalised to body weight (BW), and variables of interest were computed. The maximal GRF for each jump, GRF max $_{\text {, }}$ was determined as the highest force achieved after landing. ${ }^{24}$ The rate of change in force, $\mathrm{RF}_{\max }$, was determined by using finite differences to calculate the slope of the force-time curve and identify the peak value. Figure 1 presents exemplar data from a $30 \mathrm{~cm}$ depth jump. In the jumps that were performed in 3-5 repetitions, the mean GRF was calculated and is reported.

\section{Statistical analysis}

We report means (SD) and 95\% confidence intervals (CI) for age, height, mass, dynamic power (long jump and vertical jump) and physical activity for the whole group and by sex (table 1). We also report means (SD) for maximal force and time to maximal force for each of the 12 jumps. We used independent $t$ tests to compare $\mathrm{GRF}_{\max }$ and $\mathrm{RF}_{\max }$ between boys and girls. We compared GRFs within each type of jump using a $t$ test when there were two jumps (JJ, SS) and analysis of variance when there were three jumps (for example, $\mathrm{DJ}_{10}, \mathrm{DJ}_{30}, \mathrm{DJ}_{50}$ ). Data were analysed using SPSS for Windows, version 8.0 (SPSS Inc, Chicago, Illinois, USA). Results were considered significant if $\mathrm{p}<0.05$.

\section{RESULTS}

Of the 70 subjects, 16 girls were Tanner I, 14 were Tanner II, and four were Tanner III. Most of the boys were Tanner I $(\mathrm{n}=31)$, four were Tanner II, and one had reached Tanner stage III.

\section{$\mathrm{GRF}_{\max }$}

Table 2 presents summary biomechanical data. The mean $\mathrm{GRF}_{\max }$ for the jumps varied from about 5 times BW (mean 5.2 times BW) for PJs, the CMJ, and $\mathrm{DJ}_{50}$ to 3 and 4 times BW (mean 3.6 times BW) for $\mathrm{DJ}_{10}, \mathrm{DJ}{ }_{30}, \mathrm{SS}_{10}, \mathrm{SS}_{20}, \mathrm{JJ}_{\max }$, and $\mathrm{JJ}_{\text {submax }}$. Only the AF jumps were below 3 times $\mathrm{BW}$ (2 times BW).

The mean GRF of $\mathrm{DJ}_{50}$ was $21 \%$ greater than the mean for $\mathrm{DJ}_{30}$ and this mean was $22 \%$ greater than the mean of $\mathrm{DJ}_{10}$

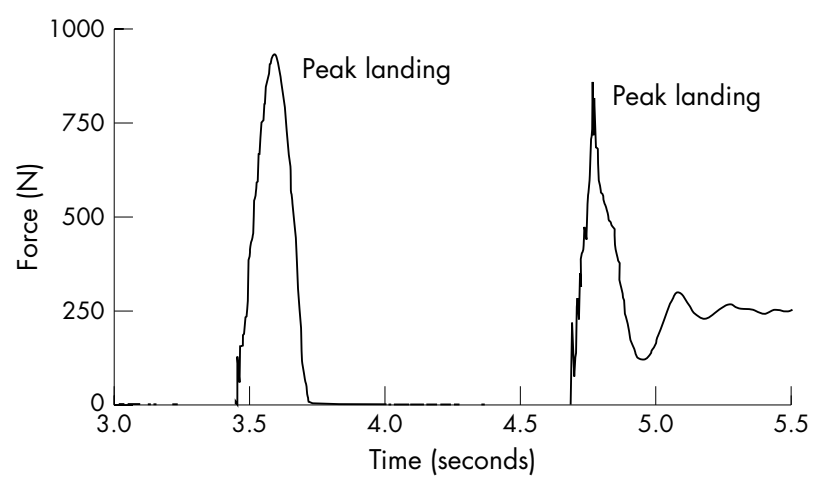

Figure 1 Exemplar force-time curve for a drop jump from a $30 \mathrm{~cm}$ platform followed by a plyometric jump. For both jumps the peak force and the rate of change in force were determined. (both $\mathrm{p}<0.05$ ). There were no significant differences in $\mathrm{GRF}_{\max }$ between the two levels of JJ and SS jumps or for the three PJs. There was, however, substantial variability in the absolute $\mathrm{GRF}_{\max }$ values for each of the jumps. For example, children could have $\mathrm{GRF}_{\max }$ of 2-7 times BW for each of JJ, SS, and DJ and 2-11 times BW for PJs.

\section{$\mathrm{RF}_{\max }$}

Maximal rates of change in force mirrored GRF outcomes. PJs and CMJs had the highest mean $\mathrm{RF}_{\max }$ values (>400 times BW/s; table 2 ). By comparison, JJ submax and AF jump had the lowest mean $\mathrm{RF}_{\max }$ values $(<200$ times $\mathrm{BW} / \mathrm{s})$. Mean values for CMJs and $\mathrm{PJ}_{\mathrm{s}}$ were significantly $(\mathrm{p}<0.05)$ greater than all other jumps, with differences as high as $30 \%$ between two foot jumps. Absolute values for $\mathrm{RF}_{\max }$ ranged from $8 \mathrm{kN} / \mathrm{s}$ (22 times $\mathrm{BW} / \mathrm{s}$ ) for $\mathrm{DJ}_{10}$ to $501 \mathrm{kN} / \mathrm{s}$ (1464 times BW/s) for a CMJ landing.

When the levels of DJs were compared, the maximal rate of force generated by $\mathrm{DJ}_{50}$ was significantly greater $(30 \%)$ than that generated by $\mathrm{DJ}_{30}$, which was significantly greater $(5 \%)$ than that generated by $\mathrm{DJ}_{10}$. The $\mathrm{RF}_{\max }$ for the $\mathrm{JJ}_{\text {submax }}$ was significantly lower $(32 \%)$ than that for the $\mathrm{JJ}_{\max }$. As expected, PJs were not significantly different from each other.

\section{Sex differences}

There were no differences in age, height, mass, and loaded physical activity between boys and girls. There were, however, sex differences for lower limb dynamic power as assessed by standing long jump. Boys jumped 14.5\% higher and $7.4 \%$ further than girls. Compared with girls, boys had greater $\mathrm{GRF}_{\max }$ for $\mathrm{CMJ}(5.9 \vee 4.7), \mathrm{PJ}_{30}(5.6 \vee 4.8)$, and $\mathrm{PJ}_{50}$ $(5.9 v 4.9)$. Boys also produced higher maximal rates of force change than girls for $J_{J_{\text {submax }}}(195 v 123), \mathrm{JJ}_{\max }(255 v 165)$, CMJ (566 v 416), $\mathrm{PJ}_{30}(538 v 336), \mathrm{PJ}_{50}(547 v 362)$, and $\mathrm{DJ}_{50}$ (452 v 320).

\section{DISCUSSION}

Although there have been numerous interventions that have augmented bone health, there is a dearth of data quantifying the impact associated with interventions. This project quantifies GRFs experienced by children while performing different types of jump during a loading intervention programme introduced as a part of elementary school physical education. This 10 minute programme of exercise elicited an osteogenic response in a multiethnic group of prepubertal and early pubertal girls and boys. ${ }^{235}{ }^{16} \mathrm{~A}$ circuit training programme comprising the measured jumping activities at five stations elicited a $2-3 \%$ greater bone accrual response at the femoral neck of the proximal femur in early pubertal girls and boys in the exercising group at eight months ${ }^{2}{ }^{17}$ and 20 months. ${ }^{2}$ Underpinning these greater bone mineral changes at the femoral neck were increased bone cross sectional area $(2 \%)$ and increased cortical thickness $(3 \%)$. Changes in these structural variables translated into a $4 \%$ greater increase in section modulus (a surrogate for bending and torsional strength). ${ }^{5}$

Key findings of this study are that maximal GRFs experienced by 9.5 year old children during jumping ranged from 2 to 5 times BW, and all two foot jumps elicited forces greater than 3.4 times BW. To our knowledge, these are the first data that quantify a range of jumping activities in this age group, and these activities can easily be incorporated into elementary school physical education. Thus the data have public health implications. Previous studies found that children experience GRF of 1 times BW while walking ${ }^{25}$ and 3 times BW during running. ${ }^{21}$ Maximal GRFs of 8.5 times BW (5.6 times BW at initial contact) have recently been reported for landings from a $61 \mathrm{~cm}$ box $(\mathrm{n}=13)$. 
Interestingly, Fuchs et al ${ }^{26}$ reported an osteotrophic response at the proximal femur that was $5 \%$ greater for the 9.3 year old children in their study who performed 100 DJ trials per exercise session, compared with controls. This compares with a $2-3 \%$ greater response from our cohort of early pubertal girls who progressed to exercises over eight months that elicited $\mathrm{GRF}_{\max }$ of 5 times BW. Because our intervention consisted of numerous jumps, it provides a physical education curriculum that maintains the child's interest but it does not allow us to identify the relative contribution of the individual jumps to this result.

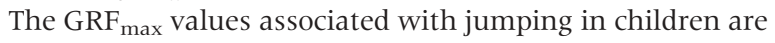
similar in magnitude to those reported in older people. Low impact aerobics (where one foot is in contact with the ground at all times) produced GRFs of 1.5 times BW, whereas high impact aerobics generated GRFs of 2-3.5 times BW. ${ }^{27}$ Extremely high landing GRFs arise in elite athletes in high impact sports. For example, gymnasts landing from the horizontal bars produce forces 8.2-11.6 times $\mathrm{BW},{ }^{28}$ and during the "step" phase elite triple jumpers generate GRFs over 15 times BW. ${ }^{29}$

In this paediatric study, the PJs and CMJs produced the highest $\mathrm{GRF}_{\max }$ and $\mathrm{RF}_{\max }$. GRF $\mathrm{Gmax}_{\max }$ values were also progressively higher when subjects dropped from 10, 30, and $50 \mathrm{~cm}$. However, this relation was not linear, which implies that the children dampened the additional kinetic energy gained by elevating the platform, probably by adapting their landing strategy. We note that, in adults, DJs from heights of $26-52 \mathrm{~cm}$ did not cause higher tibial strain or strain rates. ${ }^{11}$

During the first 150-200 milliseconds after impact there are significant variations between adult subjects in the magnitude of peak force, ${ }^{30}$ and these determined whether the landing was classified as "hard" or "soft". During hard landing, the lower legs undergo negative vertical acceleration, followed by a positive acceleration, which is attributed to muscular stabilisation. During soft landing, on the other hand, the initial negative acceleration is smaller and changes slowly to a relatively small positive acceleration. The leg musculature is absorbing the impact energy over a longer time, thus reducing the peak contact force.

\section{Role of technique in attenuating landing forces}

Ability to attenuate landing forces may be linked with skill level. Studies that compared elite and recreational athletes showed that recreational athletes flexed the hip joint to a lesser degree during landings from a low DJ and to a greater degree during landings from a high DJ than highly trained athletes. ${ }^{19}$

It has been suggested that children do not have the skill to produce a soft landing on request. In a survey of two groups of 9 and 11 year old schoolboys, none of the 20 subjects could produce a soft landing. The typical peak acceleration by the subjects was $40-60 \mathrm{~m} / \mathrm{s}^{2}$ as compared with $20-30 \mathrm{~m} / \mathrm{s}^{2}$ for adults. ${ }^{30}$ We assessed physical activity habits of these children but had no clear indication of skill level within these activities. There was no association between $\mathrm{GRF}_{\max }$ and participation in loaded physical activity or with maturity. Whether landing strategy varies with activity level, skill, or maturity in growing children warrants further study. In one study the average GRFs associated with $61 \mathrm{~cm}$ box jumps did not change significantly across 100 trials. ${ }^{15}$

Those authors ${ }^{15}$ reported maximal GRFs of 8.5 times BW whereas the children in the present study landed with GRFs approximately 4.7 times BW. It is most unlikely that the height of the platform alone would explain this difference. Thus we speculate that differences in the age, size, or skill level of the children between studies may account for these substantial differences. Although not addressed in this study, a closer look at the determinants of GRF (and rate of force change) in children is of interest. In adults, muscular and kinetic shock absorbing mechanisms during drop jumping may explain a lower than expected strain rate. ${ }^{11} 31$

The force-time curves illustrate some of the differences in landings between jumps. During fast, repeated jumping the landing and take off fuse, as the action is fast enough to exploit the absorption of elastic energy that occurs during landing and is reused during take off. ${ }^{32}$ The repeated jumpsfor example, JJ and SS - of this study were ballistic in nature, and the force-time curves show that subjects landed and pushed off very rapidly. The short time period available to begin the next jump may have prevented subjects from "catching" themselves and softening the landing. On the other hand, for the repeated maximal jumps, landing forces would be attenuated if the subjects flexed their knees in preparation for the next jump. A kinematic study of landing strategies would elucidate these differences.

Although an important and clinically relevant question, our study design did not allow us to calculate the strain experienced at the proximal femur. In kinematic studies, forces at the feet transmitted up along the lower limb to the hip, where they approximated 10 times BW. ${ }^{33}$ In an adult study where strain was measured in situ from gauges embedded in hip prostheses, implant forces at the hip were 2.5-3.0 times the GRFs during take off, and 1.5 times the GRFs during landing, from a jump. ${ }^{13}$

The greater forces were attributed to the contraction of the large extensor muscles of the knee that attach across the femur and apply a compressive force to the shaft. In a similar study, walking at $1 \mathrm{~km} / \mathrm{h}$ produced forces 2.8 times BW at the hip and 4.8 times BW when walking at $5 \mathrm{~km} / \mathrm{h}$. Jogging elicited hip forces approximating 5.5 times BW. ${ }^{34}$ In addition, Bauer $e t ~ a l^{15}$ estimated hip reaction forces in children at first peak using a simple rigid body model (without the contribution of muscular forces) to be 4.2 times BW.

In this study, boys generated significantly higher $\mathrm{GRF}_{\max }$ and $\mathrm{RF}_{\max }$ (JJs, CMJ, and DJs) than girls. For measures of dynamic power, boys also performed significantly better than girls. This performance advantage is not explained by body composition differences, as height, sitting height, mass, and the amount of lean and fat mass were similar between boys and girls. Boys and girls also engaged in similar amounts of physical activity. We do not know, however, whether the boys engaged in more jumping activities specific to this investigation than girls. Before puberty, boys generally outperform girls when jumping. ${ }^{35}$ Sex related differences in motor performance are increasingly apparent as children approach adolescence, ${ }^{35}$ but we were somewhat surprised by the sex differences in these prepubertal and early pubertal children.

\section{What is already known on this topic}

Common jumping activities in children produce GRFs from 2 to over 5 times body weight. Forces can be attenuated with landing strategies when jumps from increased heights are adopted as a part of "progressive" exercise interventions.

\section{What this study adds}

The jumps tested in this study led to positive change in numerous measures of bone health (which are reported elsewhere ${ }^{216}$ ). 
A limitation of the study was that seven of the 12 jumping activities were not measured as repeated activities-that is, three jumps with a mean reported. This was a practical limitation because of time, as children were coming to the laboratory during the school day in a bus, undertaking three practices for each jumping activity, and completing 12 different jumps. Future studies with fewer children could address this limitation.

In summary, we observed $\mathrm{GRF}_{\max }$ from 2 to over 5 times BW in children performing 12 varied jumping activities. The activities that had $\mathrm{GRF}_{\max }$ greater than 5 times BW were the PJ and the CMJ. Our data show that, as in adults, ${ }^{11}$ children appear to use landing strategies to attenuate GRFs when jumps from increased heights form part of "progressive" exercise interventions. However, extrapolating from animal studies, it would appear likely that even a small number of jump repetitions ${ }^{36}$ that provide high strain rate may benefit bone health. ${ }^{37}$ Future biomechanical studies may focus on ways of measuring or estimating strain rate at various skeletal sites associated with specific exercises.

\section{ACKNOWLEDGEMENTS}

We are grateful to the children, parents, teachers, and principals in the Richmond School District for their participation. Thanks go to Thomas R Oxland for his review of this manuscript. This research project was supported by the Michael Smith Foundation for Health Research. HMcK is a Michael Smith Foundation for Health Research Senior Scholar, and KMK is a Canadian Institutes of Health Research New Investigator (Institute of Musculoskeletal Health and Arthritis).

\section{Authors' affiliations}

H McKay, Department of Family Practice and Orthopaedics, Faculty of Medicine, University of British Columbia, Vancouver, Canada

G Tsang, D Sanderson, K M Khan, School of Human Kinetics, Faculty of Education, University of British Columbia

A Heinonen, University of Jyvaskyla, Jyvaskyla, Finland

K MacKelvie, BC Children's and Women's Hospital, Vancouver

Conflict of interests: none declared

\section{REFERENCES}

Bailey DA, McKay HA, Mirwald RA, et al. The University of Saskatchewan Bone Mineral Accrual Study: a six-year longitudinal study of the relationship of physical activity to bone mineral accrual in growing children. J Bone Miner Res 1999; 14:1672-9.

2 MacKelvie KJ, Khan KM, Petit MA, et al. A school-based exercise intervention elicits substantial bone health benefits: a 2 -year randomized controlled trial in girls. Paediatrics 2003;1 12:447-52.

3 McKay HA, Petit MA, Schutz RW, et al. Augmented trochanteric bone mineral density after modified physical education classes: a randomized school-based exercise intervention in prepubertal and early-pubertal children. J Pediatr 2000; 136:156-62

4 Khan K, McKay HA, Haapasalo H, et al. Does childhood and adolescence provide a unique opportunity for exercise to strengthen the skeleton? J Sci Med Sport 2000;3:150-64

5 Petit MA, McKay HA, MacKelvie KJ, et al. A randomized school-based jumping intervention confers site and maturity-specific benefits on bone structural properties in girls: a hip structural analysis study. J Bone Miner Res 2002;17:363-72.

6 Raab DM, Smith EL, Crenshaw TD, et al. Bone mechanical properties after exercise training in young and old rats. J Appl Physiol 1990;68:130-4.

7 Forwood MR, Parker AW. Effect of exercise on bone growth: mechanical and physical properties studied in the rat. Clin Biomech 1987;2:185-90.

8 Bennell K, Page C, Khan K, et al. Effects of resistance training on bone parameters in young and mature rats. Clin Exp Pharmacol Physiol 2000;27:88-94.
9 Bennell KL, Khan KM, Warmington S, et al. Age does not influence the bone response to treadmill exercise in female rats. Med Sci Sports Exerc 2002;34:1958-65.

10 Judex S, Zernicke RF. High-impact exercise and growing bone: relation between high strain rates and enhanced bone formation. J Appl Physiol 2000;88:2183-91

11 Milgrom C, Finestone A, Levi Y, et al. Do high impact exercises produce higher tibial strains than running? Br J Sports Med 2000;34:195-9.

12 Burr D, Milgrom C, Fyhrie D. In vivo measurement of human tibial strains during vigorous activity. Bone 1996;18:405-10.

13 Bassey EJ, Littlewood JJ, Taylor SJ. Relations between compressive axial forces in an instrumented massive femoral implant, ground reaction forces, and integrated electromyographs from vastus lateralis during various 'osteogenic' exercises. J Biomech 1997;30:213-23.

14 Ekenman I, Halvorsen K, Westblad P, et al. Local bone deformation at two predominant sites for stress fractures of the tibia: an in vivo study. Foot Ankle Int 1998;19:479-84.

15 Bauer J, Fuchs R, Smith G, et al. Quantifying force magnitude and loading rate from drop landings that induce osteogenesis. J Appl Biomech 2001;17:142-52.

16 MacKelvie KJ, McKay HA, Petit MA, et al. Bone mineral response to a 7 month randomized controlled, school-based jumping intervention in 121 prepubertal boys: associations with ethnicity and body mass index. J Bone Miner Res 2002;17:834-44.

17 MacKelvie KJ, McKay HA, Khan KM, et al. A school-based exercise intervention augments bone mineral accrual in early pubertal girls. J Pediatr 2001; 139:501-7

18 McKay HA, Petit M, Schutz R, et al. Lifestyle determinants of bone mineral: a comparison between prepubertal Asian- and Caucasian-Canadian boys and girls. Calcif Tissue Int 2000;66:320-4.

19 McNitt-Gray JL. Kinematics and impulse characteristics of drop landings from three heights. Int J Sport Biomech 1991;7:201-24.

20 Tanner JM. Growth and maturation during adolescence. Nutr Rev $1981 ; 39: 43-55$.

21 Engsberg JR, Lee AG, Patterson JL, et al. External loading comparisons between able-bodied and below-knee-amputee children during walking. Arch Phys Med Rehabil 1991;72:657-61.

22 Matsudo S, Matsudo V. Validity of self-evaluation on determination of sexual maturational level. In: Claessens A, Lefevre J, Eynde B, eds. Worldwide variation in physical fitness. Leuven: Institute of Physical Education, 1993.

23 AAHPERD. Youth fitness test manual: American Alliance for Health, Physical Education, Recreation and Dance. Reston, VA: AAHPERD, 1976.

24 Crossley K, Bennell KL, Wrigley T, et al. Ground reaction forces, bone characteristics, and tibial stress fracture in male runners. Med Sci Sports Exerc 1999;31:1088-93.

25 Beck RJ, Andriacchi TP, Kuo KN, et al. Changes in the gait patterns of growing children. J Bone Joint Surg [Am] 1981;63:1452-7.

26 Fuchs RK, Bauer JJ, Snow CM. Jumping improves hip and lumbar spine bone mass in prepubescent children: a randomized controlled trial. J Bone Miner Res 2001;16:148-56.

27 Michaud TJ, Rodriguez-Zayas J, Armstrong C, et al. Ground reaction forces in high impact and low impact aerobic dance. J Sports Med Phys Fitness 1993;33:359-66

28 Ozguven HN, Berme N. An experimental and analytical study of impact forces during human jumping. J Biomech 1988;21:1061-6.

29 Perttunen JO, Kyrolainen H, Komi PV, et al. Biomechanical loading in the triple jump. J Sports Sci 2000;18:363-70.

30 Lees A. Methods of impact force absorption when landing from a jump. Eng Med 1980:40:653-63.

31 Wosk J, Voloshin A. Wave attenuation in skeletons of young healthy persons. J Biomech $1981 ; 14: 261-7$

32 McNitt-Gray JL. Kinetics of the lower extremities during drop landings from three heights. J Biomech 1993;26:1037-46.

33 Burdett R. Forces predicted at the ankle during running. Med Sci Sports Exerc 1992; 14:308-18.

34 Bergmann G, Graichen F, Rohlmann A. Hip joint loading during walking and running, measured in two patients. J Biomech 1993;26:969-90.

35 Malina RM. Physical growth and biological maturation of young athletes. In: Holloszy JO, ed. Exercise and sports science reviews. Baltimore: Williams \& Wilkins, 1994:389-433.

36 Umemura Y, Ishhiko T, Yamauchi T, et al. Five jumps per day increase bone mass and breaking force in rats. J Bone Miner Res 1997; 12:1480-5.

37 Khan K, McKay H, Kannus P, et al. Physical activity and bone health. Human Kinetics 2001:23-34. 